Louisiana State University

LSU Digital Commons

Faculty Publications

Department of Physics \& Astronomy

$1-1-2015$

\title{
Multiphoton transitions for robust delay-zero calibration in attosecond transient absorption
}

\author{
J. Herrmann \\ ETH Zürich \\ M. Lucchini \\ ETH Zürich
}

S. Chen

Louisiana State University

M. Wu

Louisiana State University

A. Ludwig

ETH Zürich

See next page for additional authors

Follow this and additional works at: https://digitalcommons.Isu.edu/physics_astronomy_pubs

\section{Recommended Citation}

Herrmann, J., Lucchini, M., Chen, S., Wu, M., Ludwig, A., Kasmi, L., Schafer, K., Gallmann, L., Gallmann, L., Gaarde, M., \& Keller, U. (2015). Multiphoton transitions for robust delay-zero calibration in attosecond transient absorption. Springer Proceedings in Physics, 162, 83-86. https://doi.org/10.1007/ 978-3-319-13242-6_20

This Conference Proceeding is brought to you for free and open access by the Department of Physics \& Astronomy at LSU Digital Commons. It has been accepted for inclusion in Faculty Publications by an authorized administrator of LSU Digital Commons. For more information, please contact ir@lsu.edu. 


\section{Authors}

J. Herrmann, M. Lucchini, S. Chen, M. Wu, A. Ludwig, L. Kasmi, K. J. Schafer, L. Gallmann, L. Gallmann, M. B. Gaarde, and U. Keller 


\title{
Multiphoton Transitions for Robust Delay-Zero Calibration in Attosecond Transient Absorption
}

\author{
J. Herrmann, M. Lucchini, S. Chen, M. Wu, A. Ludwig, L. Kasmi, \\ K.J. Schafer, L. Gallmann, M.B. Gaarde and U. Keller
}

\section{Introduction}

Attosecond transient absorption spectroscopy plays a major role in the progress of attosecond science. A manifold of recent publications, either employing a single attosecond pulse (SAP) or a train of attosecond pulses (APT) in combination with an infrared (IR) pulse, provided detailed insight into the electron dynamics of atoms [1-4]. Due to the richness of the observed effects the correct calibration of the delay-zero in the experimental data is crucial for a useful interpretation.

Unfortunately, the low photon flux in the extreme ultraviolet (XUV) spectral region in combination with the absence of a suitable nonlinear material prevents the delay-zero calibration with a simple cross-correlation like in femtosecond pumpprobe spectroscopy. Furthermore, the extraction of the delay-zero out of the experimental data by simply using e.g. the total absorption leads to incorrect results.

Here, we show the transient absorption of an APT overlapped with a moderately strong IR pulse around the first ionization threshold of helium (He). In the transmitted XUV radiation as a function of APT-IR delay we observe half- and quarterlaser-cycle oscillations. The appearance of the quarter-laser-cycle $(4 \omega)$ oscillations

\footnotetext{
J. Herrmann ( $)$ · M. Lucchini · A. Ludwig · L. Kasmi · L. Gallmann · U. Keller Department of Physics, Institute for Quantum Electronics, ETH Zurich, 8093 Zurich, Switzerland e-mail: jens.herrmann@phys.ethz.ch

L. Gallmann

Institute of Applied Physics, University of Bern, 3012 Bern, Switzerland

S. Chen · M. Wu · K.J. Schafer · M.B. Gaarde

Department of Physics and Astronomy, Louisiana State University, Baton Rouge, LA 70803, USA 
allows us to experimentally define a precise and robust delay-zero. Our experimental results are supported by the solution of the time-dependent Schrödinger equation (TDSE).

\section{Results}

For the generation of the APT we use a Ti:sapphire-based laser amplifier system (repetition rate: $1 \mathrm{kHz}$, pulse duration: $\approx 25 \mathrm{fs}$, center wavelength: $789 \mathrm{~nm}$ ) which is focused into a xenon gas jet. We separate a small fraction of the IR beam before the generation of the APT and send it on an independent beampath where we can introduce a delay with respect to the APT. After recombining APT and IR pulse, we focus both beams into a He gas target and detect the transmitted XUV radiation after filtering the residual IR radiation. The APT consists of higher-order harmonics (HHs) which are energetically centered around the first ionization threshold of He. The optical density of the He gas target is 0.79 for $\mathrm{HH} 17$.

Figure 1a shows color-coded the transmitted XUV intensity as a function of photon energy and APT-IR delay $\tau$. HHs 13 to 17 exhibit strong half-laser-cycle $(2 \omega)$ oscillations. For a more detailed analysis we calculate the energy integrated absorption $\Pi(\tau)$. Figure $1 \mathrm{~b}$ depicts $\Pi(\tau)$ for $\mathrm{HH}$ 13. In order to disentangle the different oscillation components we perform a delay-frequency analysis with a Gaussian-Wigner-transform into the frequency-delay domain (see Fig. 1c). This representation exhibits which frequency component appears at which APT-IR delay. Besides the strong $2 \omega$-oscillation component at $0.76 \mathrm{PHz}$ Fig. 1c reveals a $4 \omega$-oscillation component at $1.52 \mathrm{PHz}$. These $4 \omega$-oscillations were predicted theoretically by Chen et al. [5]. By integrating the frequency-delay representation along the frequency axis around $1.52 \mathrm{PHz}$ we get the envelope of the $4 \omega$-oscillations as a function of delay as shown in Fig. 1e for an IR intensity of $2.6 \cdot 10^{14}$ $\mathrm{W} / \mathrm{cm}^{2}$. This envelope has a symmetric shape with respect to the delay. The solution of the TDSE shows that the maximum of the envelope coincides with the delay-zero and is robust against changes of the gas density in the target and changes of the IR intensity. Figure 1f presents the calculated envelope for an intensity of $2.8 \cdot 10^{14} \mathrm{~W} / \mathrm{cm}^{2}$.

\section{Conclusion and Outlook}

We studied the interaction of an APT overlapped with a moderately strong IR pulse in He by means of attosecond transient absorption spectroscopy. A delay-frequency analysis allows us to identify $4 \omega$-oscillations besides the already well-known $2 \omega$-oscillations. The $4 \omega$-oscillations originate from a multiphoton coupling of different HHs of the APT. Furthermore, we discussed how to use the $4 \omega$-oscillations to 

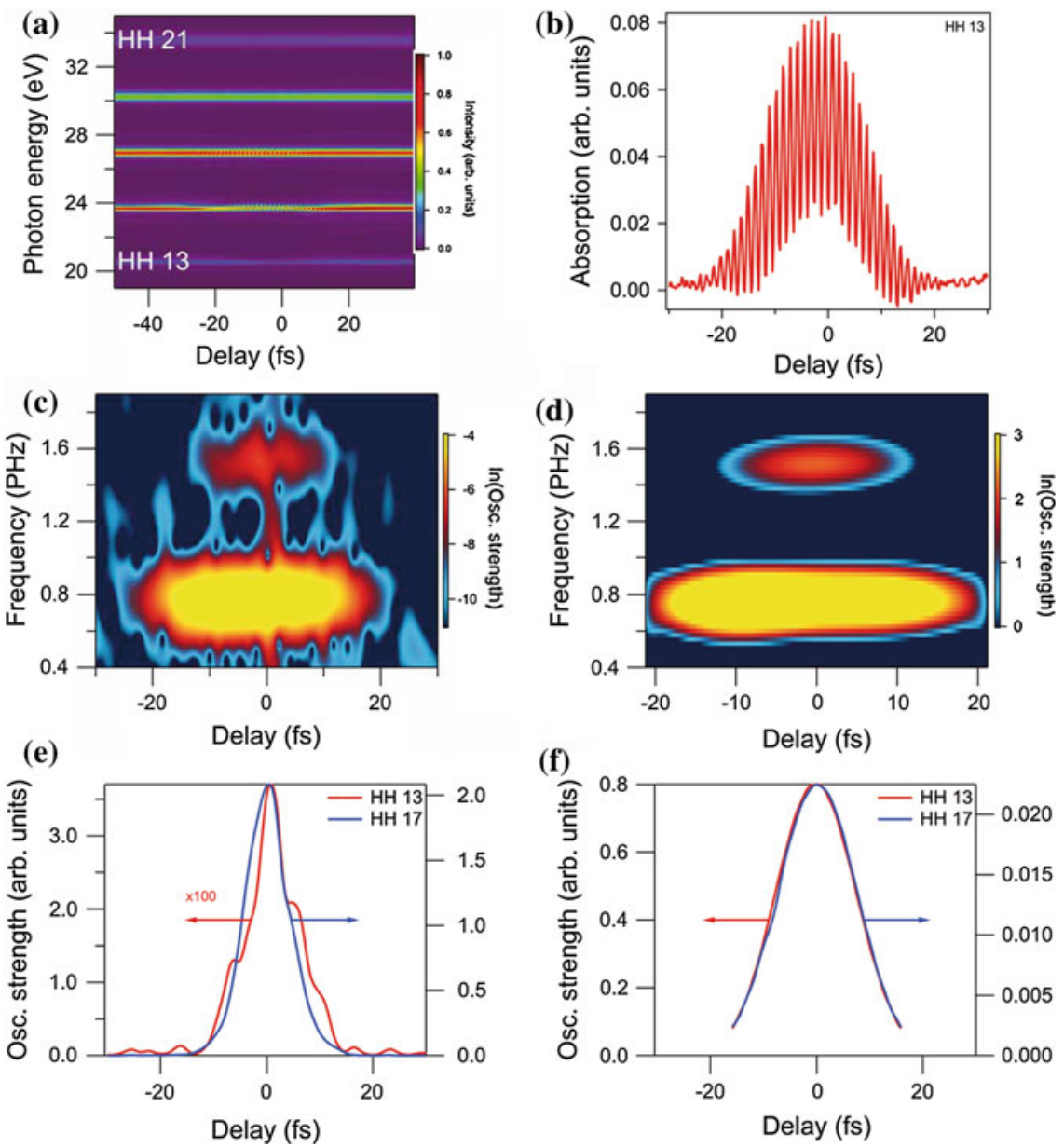

Fig. 1 a Transmitted XUV intensity as a unction of APT/IR delay and photon energy at an IR intensity of $2.6 \cdot 10^{14} \mathrm{~W} / \mathrm{cm}^{2}$. b Energy-integrated absorption of $\mathrm{HH}$ 13. c Gaussian-Wigner transform of the energy-integrated absorption of $\mathrm{HH} 13$ shown in (b). d Gaussian-Wigner transform of the calculated atomic response function. e Integration of the delay-frequency representation in the frequency domain around $1.52 \mathrm{PHz}$ yielding the envelope of $4 \omega$-oscillations of the experimental data in (c). $\mathbf{f}$ Envelope of the $4 \omega$-oscillations calculated by solving the TDSE. The peak of the oscillations coincides with the delay-zero

robustly calibrate the delay-zero in an attosecond transient absorption experiment. By solving the TDSE we support our experimental findings. Finally, we discuss in detail the influence of the IR intensity on the sub-laser-cycle oscillations. 


\section{References}

1. E. Goulielmakis, Z.-H. Loh, A. Wirth, R. Santra, N. Rohringer, V. S. Yakovlev, S. Zherebtsov, T. Pfeifer, A. M. Azzeer, M. F. Kling, S. R. Leone, and F. Krausz, Nature 466, 739-743 (2010). 2. M. Holler, F. Schapper, L. Gallmann, and U. Keller, Phys. Rev. Lett. 106, 123601 (2011).

3. M. Lucchini, J. Herrmann, A. Ludwig, R. Locher, M. Sabbar, L. Gallmann, and U. Keller, New J. Phys. 15, 15 (2013).

4. M. Chini, X. Wang, Y. Cheng, Y. Wu, D. Zhao, D. A. Telnov, S.-I. Chu, and Z. Chang, Sci. Rep. 3, 1105 (2013).

5. S. Chen, K. J. Schafer, and M. B. Gaarde, Opt. Lett. 37, 2211-2213 (2012). 